\title{
O impacto da pandemia de COVID-19 no trabalho dos profissionais da saúde: Uma
}

\section{Revisão Integrativa}

\author{
The pandemic impact of COVID-19 on the work of health professionals: An Integrative \\ El impacto de la pandemia COVID-19 en el trabajo de los profesionales de la salud: una revisión
}

integradora

Recebido: 15/06/2021 | Revisado: 21/06/2021 | Aceito: 22/06/2021 | Publicado: 09/07/2021

\author{
Grazieli dos Santos Araripe \\ ORCID: https://orcid.org/0000-0002-3391-4154 \\ Faculdade de Ensino Superior do Piaú, Brasil \\ E-mail: grazyararipe@gmail.com \\ Gislene Mariana Pereira Castelo Branco \\ ORCID: https://orcid.org/0000-0003-4393-771X \\ Faculdade de Ensino Superior do Piauí, Brasil \\ E-mail:gi.marianna@hotmail.com \\ Ruth Raquel Soares de Farias \\ ORCID: https://orcid.org/0000-0002-0988-0900 \\ Faculdade de Ensino Superior do Piauí, Brasil \\ E-mail: ruthraquelsf@gmail.com
}

\begin{abstract}
Resumo
Por conta da fácil disseminação e infecção, a COVID-19 criou um delicado cenário de pandemia que influenciou diretamente e indiretamente diversos setores da sociedade. Entre estes setores, os hospitais se tornaram ambientes de trabalho especialmente delicados devido à dificuldade para prevenção e tratamento da doença. Partindo da hipótese de que o desgaste físico e emocional causado pela intensificação da jornada de trabalho no período de pandemia contribua para o esgotamento físico e emocional, o presente artigo possui o objetivo de apontar os impactos da pandemia de COVID-19 no trabalho dos profissionais da saúde. Para isso, uma revisão integrativa foi feita a partir da consulta de textos encontrados no portal de periódicos BVS (Biblioteca Virtual em Saúde) utilizando as bases de dados: LILACS (Literatura Latino-Americana e do Caribe em Ciências da Saúde), MEDLINE e IBECS (Índice Bibliográfico Español en Ciencias de la Salud). Nesta revisão, 9 artigos foram incluídos e lidos integralmente. Através desta pesquisa pode ser percebido que profissionais da saúde tem o risco cotidiano ampliado por conta dos perigos gerados pelo cenário de pandemia. A pressão do trabalho em circunstâncias delicadas resulta em estresse físico e emocional podendo resultar em problemas de ansiedade e prejudicar a qualidade do trabalho oferecida por estes profissionais. A melhoria de fatores como infraestrutura e mudança na rotina de trabalho, pode diminuir estes problemas e auxiliar na melhora do bem estar profissional.
\end{abstract}

Palavras-chave: Pandemia; COVID-19; Profissionais da saúde.

\begin{abstract}
Due to its easy dissemination and infection, COVID-19 created a delicate pandemic scenario that directly and indirectly influenced various sectors of society. Among these sectors, hospitals have become especially delicate work environments due to the difficulty in preventing and treating the disease. Assuming that the physical and emotional exhaustion caused by the intensification of working hours during the pandemic period contributes to physical and emotional exhaustion, this article aims to point out the impacts of the COVID-19 pandemic on the work of professionals from health. For this, an integrative review was carried out based on the consultation of texts found in the portal of journals VHL (Virtual Health Library) using the databases: LILACS (Latin American and Caribbean Literature in Health Sciences), MEDLINE and IBECS (Bibliographic Index Español en Ciencias de la Salud). In this review, 9 articles were included and read in full. Through this research, it can be seen that health professionals have increased daily risk due to the dangers generated by the pandemic scenario. The pressure of work in delicate circumstances results in physical and emotional stress, which can result in anxiety problems and impair the quality of work offered by these professionals. The improvement of factors such as infrastructure can reduce these problems and help improve professional well-being.
\end{abstract}

Keywords: Pandemic; COVID-19; Health professionals. 


\begin{abstract}
Resumen
Debido a la fácil diseminación e infección, COVID-19 creó un delicado escenario pandémico que influyó directa e indirectamente en varios sectores de la sociedad. Entre estos sectores, los hospitales se han convertido en entornos laborales especialmente delicados debido a la dificultad para prevenir y tratar la enfermedad. Asumiendo que el agotamiento físico y emocional que ocasiona la intensificación de la jornada laboral durante el período pandémico contribuye al agotamiento físico y emocional, este artículo tiene como objetivo señalar los impactos de la pandemia COVID-19 en el trabajo de los profesionales de la salud. Para ello, se realizó una revisión integradora a partir de la consulta de textos encontrados en el portal de revistas BVS (Virtual Health Library) utilizando las siguientes bases de datos: LILACS (Literatura Latinoamericana y Caribeña en Ciencias de la Salud), MEDLINE e IBECS (Índice Bibliográfico Español en Ciencias de la Salud). En esta revisión se incluyeron y leyeron en su totalidad 9 artículos. A través de esta investigación, se puede constatar que los profesionales de la salud han aumentado el riesgo diario debido a los peligros que genera el escenario pandémico. La presión del trabajo en circunstancias delicadas se traduce en estrés físico y emocional, que puede derivar en problemas de ansiedad y perjudicar la calidad del trabajo ofrecido por estos profesionales. La mejora de factores como la infraestructura y los cambios en la rutina laboral pueden reducir estos problemas y ayudar a mejorar el bienestar profesional.
\end{abstract}

Palabras clave: Pandemia; COVID-19; Profesionales de la salud.

\title{
1. Introdução
}

Ainda sendo estudado por conta de sua recente descoberta, o coronavírus propaga a COVID-19, uma doença com sintomas semelhantes à gripe onde o contágio ocorre quando o sujeito infectado libera gotículas de saliva, espirro ou tosse. Galvão e Silva (2020) destaca também a possibilidade de contágio através do contato direto com as membranas mucosas e do contato com uma superfície ou objeto contaminado, o que aumenta as possibilidades de infecção.

Apesar da fácil propagação, a COVID-19 é capaz de causar quadros clínicos variados. A respeito da gravidade desta doença, o Ministério da Saúde (2020, p.3) afirma que, "a COVID-19 é uma doença causada pelo coronavírus, denominada SARS-CoV-2, que apresenta um espectro clínico variando de infecções assintomáticas a quadros graves".

Deste modo, "Sabe-se que o vírus tem alta transmissibilidade e pode provocar uma síndrome respiratória aguda que varia de casos leves (cerca de 80\%), a casos muito graves com insuficiência respiratória (entre 5\% e 10\% dos casos)." (Brasil, 2020, p.9). Em quadros menos graves, persistem as semelhanças com a gripe comum.

De acordo com o Ministério da Saúde (2020) além de febre e tosse seca frequente, sintomas como fadiga, dispneia, mal estar e mialgia, sintomas respiratórios do trato superior e sintomas gastrointestinais (mais raros) também podem ocorrer. Em quadros clínicos de infecções graves, os sintomas já mencionados se mostrarem mais agressivos, um indivíduo infectado pode apresentar dificuldade de respirar, dor no peito e perda de fala ou movimento.

O Ministério da Saúde (2020) também destaca que quadros mais graves costumam ocorrer com mais frequência em adultos com idade acima de 60 caracterizando assim o principal grupo de risco, porém, a grande capacidade de transmissibilidade do vírus afeta não apenas pessoas de terceira idade.

A variedade de quadros causados pela infecção tornou necessária a criação de medidas de isolamento e distanciamento social reduzindo e consequentemente transformando o cotidiano de diversos setores da sociedade como o de eventos e o transporte público devido a possibilidade de criação de ambientes com alto risco de transmissibilidade. Entretanto, neste cenário onde ocorrem redução de carga horaria e de movimento, os profissionais de saúde tiveram sua carg a de trabalho aumentada e com isso as medidas de isolamento social não se aplicaram a este público (Teixeira et al., 2020).

Por trabalharem em hospitais que geralmente são ambientes que prestam assistência a pacientes infectados, os profissionais da saúde frequentam áreas que possuem um maior risco pois, "A transmissão de pessoa para pessoa pode ser maior em hospitais, local onde a preocupação com aerossóis é fundamental [...]” (Senhoras, p. 58, 2020).

Assim, o trabalho de profissionais da saúde se torna especialmente delicado pois: 
Os profissionais de saúde constituem um grupo de risco para a COVID-19 por estarem expostos diretamente aos pacientes infectados, o que faz com que recebam uma alta carga viral (milhões de partículas de vírus). Além disso, estão submetidos a enorme estresse ao atender esses pacientes, muitos em situação grave, em condições de trabalho, frequentemente, inadequadas. (Teixeira et al., p. 3466, 2020).

Além da dificuldade relacionada a exposição de profissionais da saúde a pacientes infectados, as constantes mudanças no fluxo de atendimentos e protocolos institucionais causam uma insegurança que dificulta a rotina de trabalho do pessoal da saúde causando pressão psicológica e agravando problemas mentais (Rodrigues \& Silva, 2020). A gravidade da doença, o medo de contrair e/ou transmitir, o estigma vivenciado pelo receio das pessoas na ida ou volta do trabalho trazem, inevitavelmente, sofrimento psíquico para os profissionais de saúde à frente dos serviços hospitalares ou comunitários (Saidel et al,2020).

Levando em conta este cenário, faz-se necessária a investigação da forma como os profissionais de saúde sentem os impactos da pandemia, especialmente na atuação na "linha de frente" no combate ao COVID-19, pois além de estarem expostos ao vírus, este grupo de profissionais tiveram sua jornada de trabalho intensificada causando assim, desgaste físico e emocional. Com isso, a intensificação do trabalho hospitalar se torna provável que não apenas por conta de complicações de saúde causadas pelo COVID-19, mas também devido a diversos outros fatores relacionados à infraestrutura, atendimento e número de profissionais que atuando que podem intensificar complicações relacionadas ao cenário de pandemia.

Portanto, partindo da hipótese que o desgaste físico e emocional causado pela intensificação da jornada de trabalho no período de pandemia contribua para o esgotamento físico e emocional, o presente texto tem como objetivo geral: Apontar os impactos da pandemia de COVID-19 no trabalho dos profissionais da saúde.

\section{Metodologia}

Este trabalho é uma revisão integrativa baseada na pesquisa de artigos publicados com a temática relacionada ao trabalho de profissionais no período de pandemia. A metodologia proposta para a pesquisa leva em conta os procedimentos adequados para a realização de uma revisão integrativa. Sousa, Silva e Carvalho (2010, p.103) afirmam que:

A revisão integrativa, [...], é a mais ampla abordagem metodológica referente às revisões, permitindo a inclusão de estudos experimentais e não-experimentais para uma compreensão completa do fenômeno analisado. Combina também dados da literatura teórica e empírica, além de incorporar um vasto leque de propósitos: definição de conceitos, revisão de teorias e evidências, e análise de problemas metodológicos de um tópico particular.

Para este estudo, a seguinte problemática procura ser respondida: Como o cenário de pandemia afeta o trabalho dos profissionais da saúde? Para compreender como as transformações causadas pela pandemia de COVID-19 no trabalho hospitalar influenciam o profissional da saúde, uma pesquisa foi feita a partir da consulta de publicações encontrados no portal de periódicos BVS (Biblioteca Virtual em Saúde) utilizando as bases de dados: LILACS (Literatura Latino-Americana e do Caribe em Ciências da Saúde), MEDLINE e IBECS (Índice Bibliográfico Español en Ciencias de la Salud). A busca foi realizada por meio de acesso online em maio de 2021, com os seguintes descritores em ciência e saúde (DeCS): "COVID-19", "Condições de Saúde" e "Pessoal de Saúde" e o operador booleano "AND".

Os critérios de inclusão estabelecidos para a pesquisa foram: artigos originais, completos, publicados entre os anos de 2019 e 2021, no idioma português, inglês e espanhol que respondiam à problemática da pesquisa. Foram excluídos monografias, dissertações, teses, artigos não estão disponíveis na íntegra, e não gratuitos.

A organização das informações encontradas nos artigos foi realizada através de um quadro (Quadro I) organizado por colunas que se referem ao título do artigo, autores, tipo de estudo, objetivos, principais conclusões, ano e país. A triagem dos artigos foi realizada a partir da consulta do título dos artigos, leitura dos resumos e por fim leitura do texto completo. 


\section{Resultados}

Entre os 22 artigos encontrados, 8 foram excluídos após a leitura de seus títulos e resumos que demonstravam afastamento do tema proposto pela presente pesquisa restando 12 artigos para serem lidos. Entre estes, 1 não forneceu o acesso ao texto completo e 2 foram excluídos por temática restando. O fluxograma a seguir ilustra o processo de inclusão dos artigos encontrados.

Figura 1 - Fluxograma de seleção dos artigos incluídos na revisão integrativa proposta

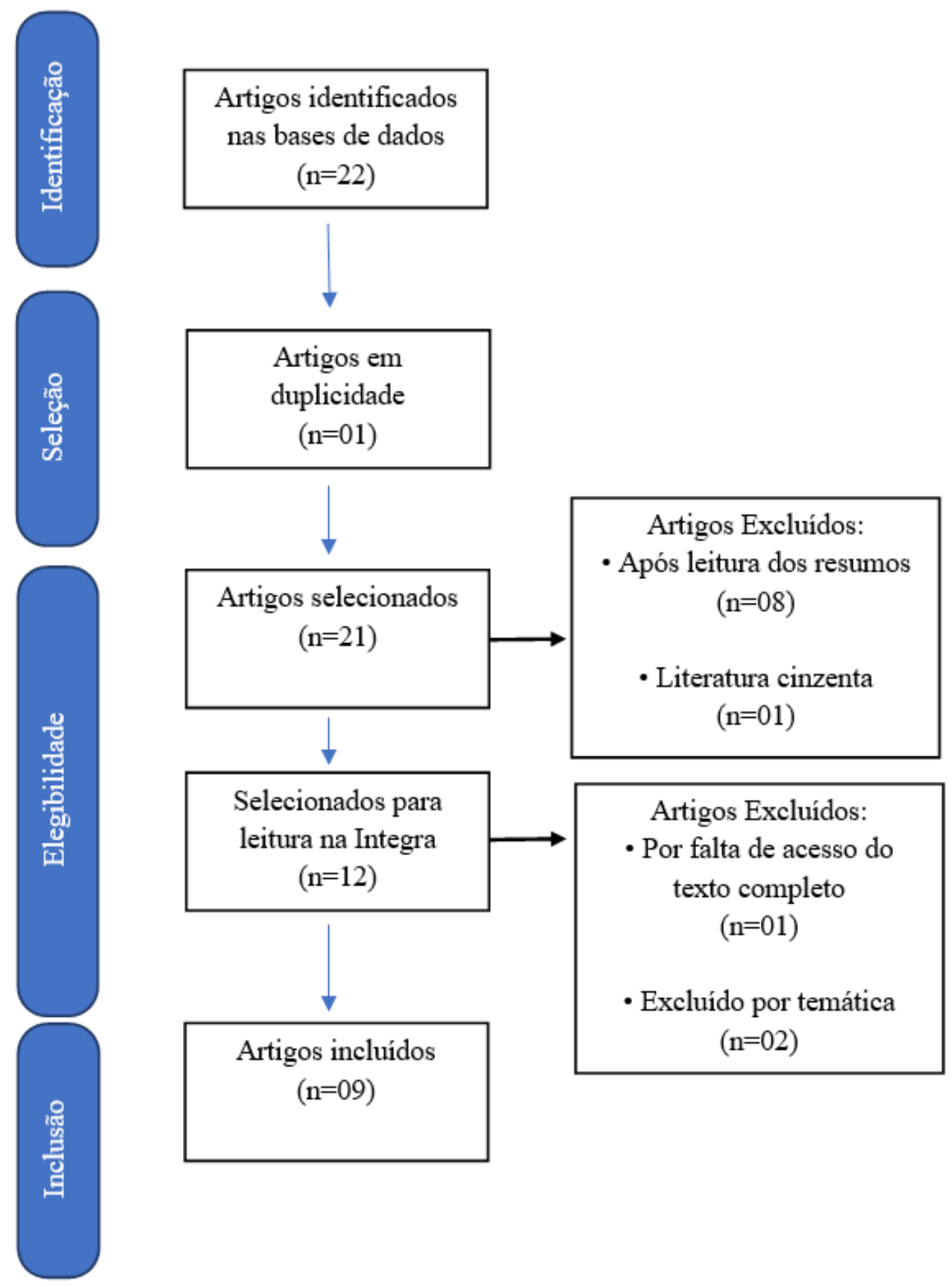

Fonte: Autores.

Observe na Figura 1 que dos 22 trabalhos encontrados na plataforma, 9 artigos atendem aos critérios estabelecidos. No Quadro 1 são apresentados os artigos incluídos na revisão integrativa, bem como são apresentados de forma sistematizada detalhes a respeito do teor de cada artigo: 


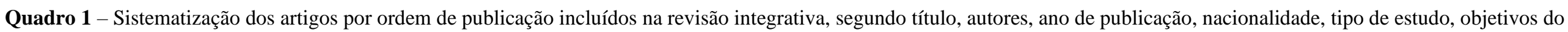
estudo e principais conclusões apresentadas.

\begin{tabular}{|c|c|c|c|c|c|c|}
\hline $\mathrm{N}^{\mathrm{o}}$ & Título & Autores & País & Tipo de estudo & Objetivo(s) & Principais Conclusões \\
\hline 1 & $\begin{array}{l}\text { Mental Health Status of } \\
\text { Healthcare Professionals and } \\
\text { Students of Health Sciences } \\
\text { Faculties in Kuwait during } \\
\text { the COVID-19 Pandemic. }\end{array}$ & $\begin{array}{l}\text { Alsairafi et. } \\
\text { al. }(2021)\end{array}$ & Suíça & $\begin{array}{l}\text { - Preferência do paciente } \\
\text { - Estudo de prevalência } \\
\text { - Fatores de risco }\end{array}$ & $\begin{array}{l}\text { Avaliar o impacto da pandemia } \\
\text { COVID-19 no estado de saúde } \\
\text { mental de profissionais de saúde e } \\
\text { estudantes de graduação em um } \\
\text { Centro de Ciências da Saúde. }\end{array}$ & $\begin{array}{l}\text { São necessários esforços proativos para apoiar sua } \\
\text { saúde mental e bem-estar por meio de campanhas } \\
\text { educacionais e programa de apoio psicológico; }\end{array}$ \\
\hline 2 & $\begin{array}{l}\text { Impact on physical and } \\
\text { mental health among medical } \\
\text { personnel in Wuhan during } \\
\text { COVID-19 outbreak: a } \\
\text { cluster analysis. }\end{array}$ & $\begin{array}{l}\text { Gao et. al. } \\
(2021)\end{array}$ & China & $\begin{array}{l}\text { - Preferência do paciente } \\
\text { - Estudo prognóstico } \\
\text { - Pesquisa qualitativa }\end{array}$ & $\begin{array}{l}\text { Explorar o impacto na saúde física e } \\
\text { mental status e classificar os } \\
\text { parâmetros entre médicos durante o } \\
\text { surto de COVID-19. }\end{array}$ & $\begin{array}{l}\text { A preocupação relacionada ao COVID-19 leva a } \\
\text { problemas de saúde física e mental entre os médicos. } \\
\text { Respostas e intervenções eficazes podem aliviar uma } \\
\text { série de novos sintomas físicos e mentais problemas } \\
\text { de saúde. }\end{array}$ \\
\hline 3 & $\begin{array}{l}\text { Working conditions and } \\
\text { health status of } 6,317 \text { front } \\
\text { line public health workers } \\
\text { across five provinces in } \\
\text { China during the COVID-19 } \\
\text { epidemic: a cross-sectional } \\
\text { study. }\end{array}$ & $\begin{array}{l}\mathrm{Li} \text { et. al. } \\
(2021)\end{array}$ & China & $\begin{array}{l}\text { - Determinantes sociais } \\
\text { de saúde } \\
\text { - Preferência do paciente } \\
\text { - Estudo de prevalência } \\
\text { - Estudo prognóstico } \\
\text { - Pesquisa qualitativa } \\
\text { - Fatores de risco }\end{array}$ & $\begin{array}{l}\text { Abordar as seguintes questões } \\
\text { relativas à mobilização e uso eficazes } \\
\text { de profissionais de saúde pública: } \\
\text { (1)papéis e funções de trabalho do } \\
\text { público profissionais de saúde } \\
\text { envolvidos em esforços de contenção } \\
\text { de epidemias; } \\
\text { (2)condições de trabalho e desafios } \\
\text { associados com o trabalho deles, } \\
\text { (3)suas percepções (por exemplo, } \\
\text { suporte percebido e problemas) } \\
\text { relacionadas ao COVID-19 e } \\
\text { trabalho, } \\
\text { (4)fatores associados a seus aspectos } \\
\text { mentais e físicos saúde (por exemplo, } \\
\text { depressão, ansiedade e autoavaliação } \\
\text { da saúde). }\end{array}$ & $\begin{array}{l}\text { Os trabalhadores da saúde pública chineses fizeram } \\
\text { imensos esforços e sacrifícios pessoais para controlar } \\
\text { o COVID-19 epidemia e enfrentou o risco de } \\
\text { problemas de saúde mental. São necessários esforços } \\
\text { para melhorar as condições de trabalho e estado de } \\
\text { saúde dos trabalhadores de saúde pública e, assim, } \\
\text { manter seu moral e eficácia durante a luta contra } \\
\text { COVID-19. }\end{array}$ \\
\hline 4 & $\begin{array}{l}\text { Factors associated with } \\
\text { mental health outcomes } \\
\text { across healthcare settings in }\end{array}$ & $\begin{array}{l}\text { Alshekaili et } \\
\text { al. (2020) }\end{array}$ & Omã & $\begin{array}{l}\text { - Estudo de etiologia } \\
\text { - Preferência do paciente } \\
\text { - Estudo de prevalência }\end{array}$ & 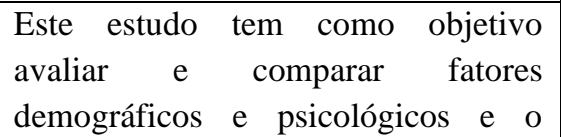 & $\begin{array}{l}\text { Este estudo sugere que os profissionais de saúde da } \\
\text { linha de frente são desproporcionalmente afetados em } \\
\text { comparação com os profissionais de saúde fora da }\end{array}$ \\
\hline
\end{tabular}




\begin{tabular}{|c|c|c|c|c|c|c|}
\hline & $\begin{array}{l}\text { Oman during COVID-19: } \\
\text { frontline versus non-frontline } \\
\text { healthcare workers. }\end{array}$ & & & - Fatores de risco & $\begin{array}{l}\text { estado do sono de profissionais de } \\
\text { saúde da linha de frente em relação } \\
\text { aos profissionais de saúde não-linha } \\
\text { de frente. }\end{array}$ & $\begin{array}{l}\text { linha de frente, com o gerenciamento dos ciclos de } \\
\text { sono-vigília e sintomas de ansiedade sendo altamente } \\
\text { endossado entre os profissionais de saúde da linha de } \\
\text { frente. }\end{array}$ \\
\hline 5 & $\begin{array}{l}\text { Assessment of Mental Health } \\
\text { Factors among Health } \\
\text { Professionals Depending on } \\
\text { Their Contact with COVID- } \\
19 \text { Patients. }\end{array}$ & $\begin{array}{l}\text { Wankowicz } \\
\text { et al. (2020) }\end{array}$ & Polônia & $\begin{array}{l}\text { - Estudo de etiologia } \\
\text { - Estudo observacional } \\
\text { - Preferência do paciente } \\
\text { - Estudo de prevalência } \\
\text { - Fatores de risco }\end{array}$ & $\begin{array}{l}\text { Avaliar os fatores de saúde mental } \\
\text { entre os profissionais de saúde, } \\
\text { quantificando a gravidade da } \\
\text { ansiedade, } \\
\text { depressão e distúrbios do sono } \\
\text { durante a atual pandemia de SARS- } \\
\text { CoV-2, levando em consideração } \\
\text { doenças coexistentes. }\end{array}$ & $\begin{array}{l}\text { Notou-se que a saúde dos trabalhadores expostos a } \\
\text { pacientes infectados com SARS-CoV-2 em } \\
\text { enfermarias de emergência, enfermarias infecciosas, } \\
\text { e unidades de terapia intensiva correm um risco } \\
\text { muito maior de apresentar sintomas de ansiedade, } \\
\text { depressão, e distúrbios do sono do que os } \\
\text { profissionais de saúde que trabalham em outras } \\
\text { enfermarias. }\end{array}$ \\
\hline 6 & $\begin{array}{l}\text { Exploring the challenges } \\
\text { faced by frontline workers in } \\
\text { health and social care amid } \\
\text { the COVID-19 pandemic: } \\
\text { experiences of frontline } \\
\text { workers in the English } \\
\text { Midlands region, UK. }\end{array}$ & $\begin{array}{l}\text { Nyashanu et } \\
\text { al. (2020) }\end{array}$ & Inglaterra & $\begin{array}{l}\text { - Determinantes sociais } \\
\text { de saúde } \\
\text { - Preferência do paciente }\end{array}$ & $\begin{array}{l}\text { Explorar os desafios enfrentados por } \\
\text { diferentes trabalhadores da linha de } \\
\text { frente em saúde e assistência social } \\
\text { durante a pandemia COVID-19. }\end{array}$ & $\begin{array}{l}\text { Os resultados do presente estudo apontam para a } \\
\text { necessidade de preparação adequada para pandemias } \\
\text { no setor de saúde e assistência social para proteger os } \\
\text { trabalhadores da linha de frente e os indivíduos que } \\
\text { cuidam }\end{array}$ \\
\hline 7 & $\begin{array}{l}\text { Study of the mental health } \\
\text { status of medical } \\
\text { personnel dealing with new } \\
\text { coronavirus } \\
\text { pneumonia }\end{array}$ & $\begin{array}{l}\text { Xing et al. } \\
(2020)\end{array}$ & $\begin{array}{l}\text { Estados } \\
\text { Unidos }\end{array}$ & $\begin{array}{l}\text { - Preferência do paciente } \\
\text { - Estudo prognóstico }\end{array}$ & $\begin{array}{l}\text { Estudar a relação entre traços de } \\
\text { personalidade e condições de saúde } \\
\text { mental do pessoal médico para } \\
\text { fornecer uma base e referência para a } \\
\text { implementação de uma educação } \\
\text { direcionada em saúde mental. }\end{array}$ & $\begin{array}{l}\text { Diante da catastrófica emergência de saúde causada } \\
\text { pela COVID-19, a equipe médica foi afetada por } \\
\text { diferentes tipos de fatores subjetivos e objetivos. } \\
\text { Seus problemas de saúde mental são uma forma de } \\
\text { resposta humana ao estresse, uma resposta } \\
\text { explicativa, emocional e defensiva dentro do corpo } \\
\text { humano e uma resposta fisiológica do corpo humano } \\
\text { à invasão de necessidades ou lesões. Nesse ambiente } \\
\text { especial, seu trabalho, vida e emoções tendem a ser } \\
\text { regularmente anormais. }\end{array}$ \\
\hline 8 & 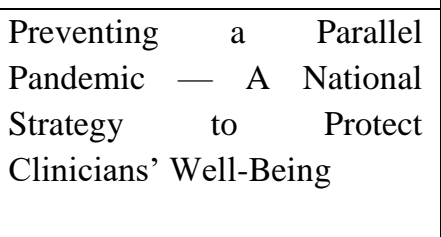 & $\begin{array}{l}\text { Dzau et al. } \\
(2020)\end{array}$ & Inglaterra & $\begin{array}{l}\text { - Determinantes sociais } \\
\text { saúde } \\
\text { - Preferência do paciente } \\
\text { - Pesquisa qualitativa }\end{array}$ & 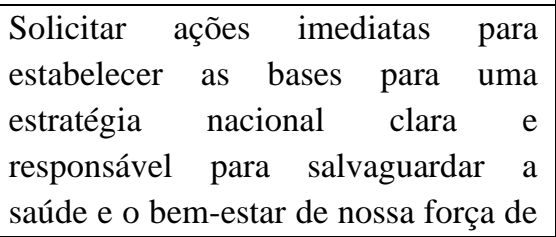 & $\begin{array}{l}\text { A crise da Covid-19 revelou com dolorosa clareza os } \\
\text { fios desgastados da força de trabalho clínica dos } \\
\text { EUA. Reparar o tecido exigirá todos nós. O bem- } \\
\text { estar do clínico é um problema complexo de sistemas } \\
\text { com várias partes responsáveis, incluindo }\end{array}$ \\
\hline
\end{tabular}


Research, Society and Development, v. 10, n. 8, e14110817210, 2021

(CC BY 4.0) | ISSN 2525-3409 | DOI: http://dx.doi.org/10.33448/rsd-v10i8.17210

\begin{tabular}{|c|l|l|l|l|l|l|}
\hline & & & & trabalho clínica. & $\begin{array}{l}\text { empregadores, } \\
\text { seguradoras, organizações de melhoria da qualidade e } \\
\text { governos estaduais e federais. }\end{array}$ \\
\hline 9 & $\begin{array}{l}\text { Coronavirus _preventing an } \\
\text { occupational hazard among } \\
\text { doctors }\end{array}$ & $\begin{array}{l}\text { Malhotra et } \\
\text { al. (2020) }\end{array}$ & Índia & $\begin{array}{l}\text { - Determinantes sociais } \\
\text { saúde } \\
\text { - Preferência do paciente }\end{array}$ & $\begin{array}{l}\text { Discutir medidas de prevenção } \\
\text { eficientes para o combate ao corona } \\
\text { vírus. }\end{array}$ & $\begin{array}{l}\text { O coquetel de alta infectividade, uma enorme } \\
\text { população suscetível, e a falta de um preventivo } \\
\text { vacina ou medicamento terapêutico trouxe uma } \\
\text { lamentável compreensão dos temores de longo prazo } \\
\text { de muitos especialistas em saúde. Nos lembrou que a } \\
\text { profissão médica precisa forçar seus limites mais } \\
\text { longe e mais rápido do que nunca para superar este } \\
\text { obstáculo. Como é o caso de qualquer pandemia, a } \\
\text { infraestrutura de saúde será colocada um caldeirão e } \\
\text { será esticado até o seu mais fino limite. }\end{array}$ \\
\hline
\end{tabular}

Fonte: Autores.

Observe que o Quadro 1 que apesar de todos os trabalhos selecionados tratarem em comum o Covid19 como temática, os tipos de estudo variam fazendo com que o tema seja analisado sob diversas perspectivas e fornecendo informações de diferentes cenários e como estes se relacionaram com a pandemia. 


\section{Discussão}

Após a leitura e seleção dos artigos, foram inclusos 9 artigos na revisão integrativa com base nos critérios estabelecidos. A análise destes tornou perceptível que todos os artigos incluídos tratam do trabalhador da saúde no período da pandemia de COVID-19, este tema permite diversos tipos de abordagem justificando a variedade dos tipos de trabalhos.

Entre os trabalhos incluídos, os artigos 1, 2, 4, 5 e 7 são trabalhos voltados para a discussão a respeito da pandemia no bem estar do profissional da saúde. Já os artigos 3, 6, 8 e 9 são trabalhos cujas discussões procuram de algum modo combater e evitar os problemas ligados a saúde mental causados pelo período de pandemia de COVID-19.

Assim, os tópicos a seguir buscam relacionar e discutir os pontos levantados nos textos inclusos na revisão integrativa.

\subsection{Influencia da pandemia de COVID-19 em profissionais da saúde}

Identificado no fim de 2019 em Wuhan na China, rapidamente o coronavírus se propagou ao redor do mundo criando uma situação de alerta. Com isso, em 31 de dezembro de 2019, a OMS declarou estado de pandemia fazendo com que diversos países adotassem medidas de isolamento afim de conter a propagação do vírus.

Independente da forma como cada nação combateu o vírus, a sua capacidade de infecção impactou diretamente os mais distintos sistemas de saúde. Apesar de não ser a primeira pandemia por qual a humanidade passa (ocorrendo anteriormente pandemias como a da peste negra e da gripe espanhola por exemplo) a pandemia de COVID-19 trazia o potencial de causar uma superlotação em hospitais por conta de seu grande poder de contágio.

De acordo com Gallasch, Cunha, Pereira e Silva-Junior (2020, p. 1), "em 11 de março de 2020 a organização mundial de saúde (OMS) caracterizou como estado de pandemia o surto mundial causado pelo novo corona vírus SARS-CoV-2, o que levou os serviços de saúde a um novo cenário de ações em saúde e segurança voltada aos diversos profissionais envolvidos no cuidado a população".

Procurando formas de minimizar a gravidade do cenário pandêmico, o setor da saúde passou por um processo de adaptação que influenciou o cotidiano dos profissionais da saúde. Após a análise minuciosa dos artigos, tornou-se perceptível que a exaustão causada pela transformação no ambiente trabalho, o aumento da quantidade de pacientes e o crescimento da carga horária tem como consequência não apenas o esgotamento físico como também o mental de profissionais da saúde "evidenciando a necessidade de medidas que visem prevenir este transtorno e que resultem em melhorias na qualidade de vida e na saúde psicológica destes trabalhadores" (Silva, Cobucci, Soares-Rachetti, Lima \& Andrade, 2021).

Observando as conclusões apresentadas pelos textos incluídos na revisão integrativa realizada, pode ser observado que existe a necessidade de melhora nas condições de trabalho dos profissionais da saúde para criar condições adequadas de trabalho evitando principalmente problemas relacionado a saúde mental dos trabalhadores deste setor.

A este respeito, Alsairafi, Naser, Alsaleh, Awad e Jalal (2021) em seu estudo destaca que a maioria dos médicos em teu trabalho tiveram contato físico com seus pacientes, o que influencia psicologicamente não só pela preocupação de contrair o vírus, mas também de transmitir para seus familiares.

Wankowicz, Szylinska, Rotter (2020) notaram que trabalhadores da saúde que atuam na linha de frente correm mais risco devido a atuação em ambientes como enfermarias de emergência, enfermarias infecciosas, e unidades de terapia intensiva e a convivência nestas áreas cria um maior risco maior de apresentar sintomas de ansiedade, depressão, e distúrbios do sono.

Sintomas como estes, de acordo com Xing, Sun, Xu, Geng e Li (2020) são uma forma de resposta humana ao estresse, uma resposta explicativa, emocional e defensiva dentro do corpo humano e uma resposta fisiológica do corpo humano à invasão de necessidades ou lesões. Deste modo, percebe-se que a repercussão da pandemia de corona vírus é maior do que a gravidade dos sintomas, da infecção e do crescente número de mortes, pois a mesma afeta a sociedade como um todo através da sua influência no sistema de saúde e dos profissionais mais próximos do combate ao vírus. 
Contudo, os profissionais de saúde constituem um grupo composto por diversas categorias que nem sempre atuam nos ambientes mencionados por Wankowicz et al. (2020). Estes profissionais também podem sofrer fisicamente e mentalmente de acordo com a forma como ocorre o gerenciamento de trabalho de acordo com Alshekaili et al. (2020), porém seu estudo aponta que os profissionais de saúde da linha de frente são desproporcionalmente afetados em comparação com os profissionais de saúde fora da linha de frente

Assim, muitos dos profissionais da saúde, por fazerem parte da linha de frente no combate a pandemia de corona vírus, ficaram sujeitos a maiores riscos não só por conta do seu contato com pacientes, mas também por conta da convivência cotidiana em um ambiente de trabalho influenciado por seu papel determinante durante o período de pandemia.

Deste modo, observa-se que a saúde mental é um fator relevante em um cenário de pandemia, podendo influenciar desde a forma como a doença é combatida até a ocorrência de sua disseminação pois mesmo que uma série de medidas sejam tomadas, muitos foram os profissionais que testaram positivo para novo corona vírus.

\subsection{Esforços para melhoria das condições de trabalho do profissional da saúde e relação com outros setores}

A série de problemas relacionados ao bem estar no trabalho e a saúde mental causados pela pandemia a profissionais da saúde torna ainda mais delicado o cenário de pandemia uma vez que a principal medida adotada para combate ao coronavírus, o isolamento social, não é uma ação na qual um profissional da saúde pra realizar de forma plena.

"Alguns profissionais de saúde infectados com o COVID-19 podem se sentir desamparados, sem esperança e isolados também. Este cenário afeta frontalmente a saúde mental dessas pessoas, necessitando que medidas protetivas desta área sejam a eles direcionadas como forma de cuidado aos cuidadores" (SANT'ANA et al, 2020)

Isso torna necessária ação não só do pessoal da saúde, mas também de outros setores da sociedade. Ao destacar os esforços pessoais dos profissionais da saúde chineses no combate ao COVID-19, Li et. al. (2021) mencionam que são necessários esforços para melhorar as condições de trabalho e estado de saúde dos trabalhadores de saúde pública e, assim, manter seu moral e eficácia durante o período de pandemia.

A este respeito Nyashanu, Pfende e Ekpenyong (2020) apontam que não só a preparação de profissionais da saúde, mas também de profissionais ligados a assistência social ganham importância no processo de proteção de trabalhadores da linha de frente e os indivíduos que cuidam.

Dzau, Kirch e Nasca (2020) ao destacarem as dificuldades do trabalho clínico nos EUA, aponta que o trabalho de combate ao covid-19 exige um trabalho complexo entre vários setores incluindo empregadores, associações profissionais, seguradoras, organizações de melhoria da qualidade e governos estaduais e federais.

Ao discutir medidas preventivas, Malhotra, Gupta e Ish (2020) destacam que a infraestrutura da saúde é um fator relevante uma vez que para a realização de um trabalho eficiente e que esta deve estar adequada para um cenário de pandemia que coloca o trabalho da saúde em no limite.

Assim, torna-se perceptível que existem ações que podem ser realizados no combate a pandemia que estão além dos esforços do pessoal da saúde e também podem auxiliar esse grupo a realizar um trabalho mais eficiente e de forma mais saudável.

\section{Conclusão}

Nesta revisão integrativa, foram incluídos 9 artigos ligados a estudos da saúde, especialmente medicina e enfermagem, que apontaram que a saúde mental de profissionais que trabalham no combate ao COVID-19 é muitas vezes afetada de forma negativa. 
A realização da pesquisa evidencia que a gravidade da pandemia torna ainda mais delicada a tarefa de profissionais da saúde. Este cenário resulta em problemas relacionados ao bem estar no trabalho e saúde mental de vários profissionais. Especialmente o que atuam na linha de frente do combate ao COVID-19 que muitas vezes precisam the dar com a exaustão física exagerada em com locais de trabalhos mais sensíveis a pandemia como enfermarias intensivas aumentado os riscos.

Também se torna perceptível a importância de outros profissionais como assistência social além de fatores como ações do governo e infraestrutura de saúde para a realização de um trabalho eficiente e para a preservação da saúde de médicos e pacientes, uma vez que o combate a COVID-19 é uma tarefa que pertence a sociedade em sua totalidade.

Assim, as mudanças na forma de trabalho e a gravidade da situação causada pelo cenário de pandemia causa não só estresse físico, mas também afeta a saúde mental gerando problemas relacionados a ansiedade, prejudicando assim profissionais e pacientes ampliando os riscos em instalações hospitalares.

Para trabalhos futuros, sugerimos uma investigação de impactos da pandemia em profissionais da saúde que atuam diretamente na recuperação dos pacientes infectados estendendo a discussão a respeito dos cuidados a serem tomados por estes durante o período de pandemia.

\section{Referências}

Alsairafi, Z., Naser, A. Y., Alsaleh, F. M., Awad, A., \& Jalal, Z. (2021). Mental Health Status of Healthcare Professionals and Students of Health Sciences Faculties in Kuwait during the COVID-19 Pandemic. Res. Public Health, 18 (4), 1-15. https://pesquisa.bvsalud.org/controlecancer/resource/pt/mdl33672372 ? src $=$ similardocs

Alshekaili, M., Hassan, W., Al Said, N., Al Sulaimani, F., Jayapal, S. K., Al-Mawali, A., Chan, M. F., Mahadevan, S., \& Al-Adawi, S. (2020). Factors associated with mental health outcomes across healthcare settings in Oman during COVID-19: frontline versus non-frontline healthcare workers. BMJ Journals, 10(10), 1-7. https://pesquisa.bvsalud.org/controlecancer/resource/pt/mdl-33672372?src=similardocs

Brasil. (2020). Sobre a doença - O que é o COVID-19. Ministério da Saúde. Secretaria de Políticas de Saúde. Brasília, DF. https://www.gov.br/saude/ptbr/vacinacao/\#: :text=O\%20que\%20é\%20COVID-19,-Os\%20coronavírus\%20são\&text=A\%20COVID-

$19 \% 20$ é\%20uma,infecções\%20assintomáticas\%20a\%20quadros\%20graves

Brasil (2020). Ministério da Saúde. Secretaria de Atenção Especializada à Saúde. Características Gerais sobre a infecção pelo novo coronavírus - SARS-COV2. In: Departamento de Atenção Hospitalar, Domiciliar e de Urgência, Protocolo de Manejo Clínico da COVID-19 na Atenção Especializada. Brasília, DF, (p.8-11) https://portalarquivos.saude.gov.br/images/pdf/2020/April/14/Protocolo-de-Manejo-Cl--nico-para-o-Covid-19.pdf.

Dzau, V. J., Kirch, D., \& Nasca, T. (2020). Preventing a Parallel Pandemic - A National Strategy to Protect Clinicians' Well-Being. The New England Journal of Medicine, 513-515. https://www.nejm.org/doi/full/10.1056/nejmp2011027.

Gallasch, C. H., Cunha, M. L., Pereira, L. A. S., \& Silva-Junior, J. S. (2020). Prevenção relacionada à exposição ocupacional: COVID-19. Revista Enfermagem UERJ, Rio de Janeiro, 28, 1-6. https://www.e-publicacoes.uerj.br/index.php/enfermagemuerj/article/view/49596.

Galvão, B. H. A., \& Silva, G. M. B (2020). Qual é o agente etiológico (causador) da covid-19?. In: PERSUHN, Darlane Camati (Orgs.). Aspectos biológicos da COVID-19: um enfoque cientifico e didático. João Pessoa: Editora UFPB. (pp. 10-11). http://www.editora.ufpb.br/sistema/press5/index.php/UFPB/catalog/download/615/723/3630-1 ?inline=1.

Gao, J., Li Jing, H. X., Yuan, Y., Li, C., \& Zhang, D. (2021) Impact on physical and mental health among medical personnel in Wuhan during COVID-19 outbreak: a cluster analysis. International Journal of Medical Sciences, 18(5), 1185-1188. https://www.medsci.org/v18p1185.htm.

Li, J., Xu, J., Zhou, H., You, H., Wang, X., Li, Y., Liang, Y., Li, S., Ma, L., Zeng, J., Cai, H., Xie, J., Pan, C., Hao, C., Gilmour, S., Tak-Fai Lau, J., Hao, Y., Roman Xu, D., \& GU, J. (2021). Working conditions and health status of 6,317 front line public health workers across five provinces in China during the COVID-19 epidemic: a cross-sectional study. BMC Public Health, 1(21), 2. https://pubmed.ncbi.nlm.nih.gov/33422035/.

Malhotra, N., Gupta, N., \& ISH, P.(2020). Coronavirus - preventing an occupational hazard among doctors. Adv Respir Med, 88(2),166-168. https://pubmed.ncbi.nlm.nih.gov/32307691/.

Nyashanu, M., Pfende, F., \& Ekpenyong, M. (2020). Exploring the challenges faced by frontline workers in health and social care amid the COVID-19 pandemic: experiences of frontline workers in the English Midlands region, UK. National Library of Medicine, 34(5), 655-661. https://pubmed.ncbi.nlm.nih.gov/32674701/.

Rodrigues, N. H., \& Silva. L. G. A. (2020) Gestão da pandemia Coronavírus em um hospital: relato de experiência profissional. Journal of Nursing and Health, 10(4), 1-9. https://periodicos.ufpel.edu.br/ojs2/index.php/enfermagem/article/view/18530/11238.

Sant'ana, G., Imoto, A. M., Amorim, F. F., Taminato, M., Peccin, M. S., Santana, L. A., Göttems, L. B. D., \& Camargo, E. B. (2020). Infecção e óbitos de profissionais da saúde por COVID-19: revisão sistemática. Acta Paulista de Enfermagem, São Paulo, 33, p. 1-9. https://www.scielo.br/scielo.php?script=sci_arttext\&pid=S0103-21002020000100601\&lng=pt\&nrm=iso\&tlng=pt\#B11.

Senhoras, E. M. (Org.) (2020). COVID-19: Enfoques preventivos. Boa Vista: Editora da Universidade Federal de Roraima. 
Research, Society and Development, v. 10, n. 8, e14110817210, 2021

(CC BY 4.0) | ISSN 2525-3409 | DOI: http://dx.doi.org/10.33448/rsd-v10i8.17210

Silva, D. F. O., Cobucci, R. N., Soares-RachettI, V. P., Lima, S. C. V. C., \& Andrade, F. B. (2021). Prevalência de ansiedade em profissionais da saúde em tempos de COVID-19: revisão sistemática com metanálise. Ciência \& Saúde Coletiva, Rio de Janeiro, RJ, 26(2), 2021. https://www.scielo.br/scielo.php?pid=S1413-81232021000200693\&script=sci_arttext\#B17.

Saidel, M. G. B., Lima, M. H. M., Campos, C. J. G., Loyola, C. M. D., Esperidião, E., \& Santos, J. R. (2020). Intervenções em saúde mental para profissionais de saúde frente a pandemia de Coronavírus. Revista enfermagem UERJ, Rio de Janeiro, 28, 1-6. https://docs.bvsalud.org/biblioref/2020/06/1097213/intervencoes-em-saude-mentalpor.pdf.

Souza, M. T., Silva, M. D., \& Carvalho, R. (2010). Revisão integrativa: o que é e como fazer. Eisntein, São Paulo, SP, 8(1),102-106. http://www.scielo.br/pdf/eins/v8n1/pt_1679-4508-eins-8-1-0102.pdf.

Teixeira, C. F. S., Soares, C. M., Souza, E. A., Lisboa, E. S., Pinto, I. C. M., Andrade, L. R., \& Espiridião, M. A. (2020). A saúde dos profissionais de saúde no enfrentamento da pandemia de Covid-19: Revista Ciência \& Saúde Coletiva da Associação Brasileira de Saúde Coletiva, Rio de Janeiro, RJ, 25(9), 34653474. https://www.scielo.br/pdf/csc/v25n9/1413-8123-csc-25-09-3465.pdf.

Wankowicz, P., Szylińska, A., \& Rotter, I. (2020). Assessment of Mental Health Factors among Health Professionals Depending on Their Contact with COVID-19 Patients. International journal of environmental research and public health, 17(16), 1-9. https://www.ncbi.nlm.nih.gov/pmc/articles/PMC7459704/.

Xing, J., Sun, N., Xu, J., Geng, S., \& Li, Y. (2020). Study of the mental health status of medical personnel dealing with new coronavirus pneumonia. PLoS ONE, 15(5), 1-10. https://journals.plos.org/plosone/article?id=10.1371/journal.pone.0233145. 